\title{
Caracterización funcional de almidones de plátano cuadrado (Musa balbisiana Colla)
}

\author{
Roxana Karina Contreras-Pérez \\ Egresada de Ingeniería en Alimentos, División Académica \\ Multidisciplinaria de los Ríos, Universidad Juárez \\ Autónoma de Tabasco, México
}

Lázaro De la Torre-Gutiérrez

Profesor de Ingeniería en Alimentos. División Académica Multidisciplinaria de los Ríos, Universidad Juárez Autónoma de Tabasco, México

\section{Nicolás González-Cortés \\ Román Jiménez-Vera}

Profesor de Ingeniería en Alimentos y Maestría en Desarrollo Alimentario Sustentable. División Académica Multidisciplinaria de los Ríos, Universidad Juárez Autónoma de Tabasco, México

Doi: 10.19044/esj.2018.v14n30p82 URL:http://dx.doi.org/10.19044/esj.2018.v14n30p82

\begin{abstract}
Resistant starch (RS) stands out for its properties related to human health. In the digestive system it provides the benefits of dietary fiber and acts as a prebiotic. New sources of resistant starch are being investigated. The proximal composition, functional properties and microbiological quality of square banana starches (Musa balbisiana Colla) were determined. The fruits were harvested in plantations of Tabasco, Mexico, with degree of maturity type I. The flour was elaborated by drying in an oven. The extraction of the starches was carried out using a wet method and the resistant starches, by enzymatic method. The concentration of bacterial groups was quantified by the surface culture method. The solubility, swelling power and water absorption patterns were evaluated at $60,70,80$ and $90^{\circ} \mathrm{C}$. Products with low moisture content were obtained: flour $(7.45 \pm 0.28)$, native starch $(9.28 \pm 0.55)$ and sterilized starch $(10.82 \pm 0.10)$. In the protein content, the highest concentration was observed in the flour ( $4.44 \pm 0.40)$, followed by the native starch $(1.19 \pm 0.04), \mathrm{RS}_{2}(0.13 \pm 0.01)$ and $\mathrm{RS}_{3}(0.19 \pm 0.01)$. Functional properties are related to different factors, such as temperature, granule size, amylose content and type of resistant starch. $\mathrm{RS}_{3}$ presented the best functional properties and good microbiological quality. The native starch presented the highest microbial load. $\mathrm{RS}_{2}$ and the sterilized starch presented the lowest values of the functional properties.
\end{abstract}


Keywords: Resistant starch, functional food, plantain, Musa balbisiana

\section{Resumen}

El almidón resistente (AR) destaca por sus propiedades relacionadas con la salud humana. En el sistema digestivo proporciona los beneficios de la fibra dietética y actúa como un prebiótico. Nuevas fuentes de almidón resistente están siendo investigadas. Se determinó la composición proximal, propiedades funcionales y calidad microbiológica de almidones de plátano cuadrado (Musa balbisiana Colla). Los frutos se recolectaron en plantaciones de Tabasco, México, con grado de madurez tipo I. La harina se elaboró mediante secado en estufa. La extracción de los almidones se realizó empleando un método húmedo y los almidones resistentes, mediante método enzimático. La concentración de grupos bacterianos se cuantificó por el método de cultivo en superficie. Los patrones de solubilidad, poder de hinchamiento y absorción de agua se evaluaron a $60,70,80$ y $90{ }^{\circ} \mathrm{C}$. Se obtuvieron productos con bajo contenido de humedad: harina $(7,45 \pm 0,28)$, almidón nativo $(9,28 \pm 0,55)$ y almidón esterilizado $(10,82 \pm 0,10)$. En el contenido de proteínas, la mayor concentración se observó en la harina $(4,44$ $\pm 0,40)$, seguido por el almidón nativo $(1,19 \pm 0,04), \mathrm{AR}_{2}(0,13 \pm 0,01)$ y $\mathrm{AR}_{3}$ $(0,19 \pm 0,01)$. Las propiedades funcionales están relacionadas con diferentes factores, como la temperatura, el tamaño del gránulo, el contenido de amilosa y el tipo de almidón resistente. $\mathrm{El} \mathrm{AR}_{3}$ presentó las mejores propiedades funcionales y buena calidad microbiológica. El almidón nativo presentó la mayor carga microbiana. El $\mathrm{AR}_{2}$ y el almidón esterilizado presentaron los valores más bajos de las propiedades funcionales.

Palabras claves: Almidón resistente, alimento funcional, plátano, Musa balbisiana

\section{Introducción}

El almidón es un carbohidrato presente en cereales, algunas raíces y legumbres. Es un polisacárido de reserva en las plantas y unos de los pocos que el hombre puede digerir. Está constituido por 2 estructuras primarias: la amilosa, un polímero lineal y la amilopectina, polímero ramificado y su principal constituyente. El almidón presenta distintos grados organizacionales, desde el nivel molecular hasta el gránulo, quienes se encuentran en los cloroplastos de las hojas verdes y en los amiloplastos de semillas, legumbres y tubérculos (Parada-S. y Rozowski-N., 2008).

El almidón es un polímero importante de origen natural con diversas aplicaciones en la ciencia de los alimentos y polímeros; la industria moderna de alimentos está aumentando la demanda del mismo por lo que se ha generado 
un interés por identificar nuevas fuentes de este polisacárido. Existe un número importante de especies vegetales que tienen un alto contenido de almidón y que podrían ser materia prima para su extracción. Dentro de ellas se encuentran algunos frutos como el plátano inmaduro, que contiene cantidades importantes de este carbohidrato, entre $70 \%$ y $80 \%$ en base seca (Blasco-López y Gómez-Montaño, 2014; Valerio-Dávila, 2014) e incluso la cáscara posee hasta un $50 \%$ de almidón en base seca (Valerio-Dávila, 2014). En un estudio realizado por Soto-Azurduy (2010), cuantificó el contenido de almidón total en 2 variedades e informó en Musa paradisiaca 73,42\% de almidón total y en Musa cavendishii, 68,13\%.

Además, el almidón destaca por sus propiedades relacionadas con la salud humana ya que una parte es indigerible, constituyendo lo que se conoce como almidón resistente (Langkilde et al., 2002; Soto-Azurduy, 2010), el cual es aprovechado por la microbiota intestinal desempeñando un papel importante en varios efectos sobre la salud humana (Lockyer y Nugent, 2017). Ha adquirido gran importancia respecto a la reducción de la respuesta glucémica, disminución de la energía consumida y la concentración de lípidos en suero, lo que favorece las condiciones fisiológicas para prevenir enfermedades como diabetes mellitus, obesidad, enfermedad cardiovascular y síndrome metabólico (Olvera-Hernández et al., 2012).

El almidón resistente se clasifica en 4 grupos: el almidón resistente tipo I $\left(\mathrm{AR}_{1}\right)$ es el almidón de difícil acceso físico, el almidón resistente tipo II $\left(\mathrm{AR}_{2}\right)$ son gránulos de almidón no gelatinizados, el almidón resistente tipo III $\left(\mathrm{AR}_{3}\right)$ es el almidón retrogradado y finalmente el almidón resistente tipo IV $\left(\mathrm{AR}_{4}\right)$ conformado por el almidón modificado químicamente, los cuales han recibido mucha atención, tanto por sus potenciales beneficios para la salud, como por sus propiedades funcionales (Rodríguez-Aguilar et al., 2014).

Desde el punto de vista funcional, el almidón constituye una excelente materia prima para modificar la textura y consistencia de los alimentos. $\mathrm{Su}$ funcionalidad está relacionada con el peso molecular de la amilosa y la amilopectina, así como de su organización molecular en el gránulo. En los alimentos regulan y estabilizan la textura, así como sus propiedades espesantes y gelificantes. Sin embargo, la estructura nativa del almidón puede reducir su uso en algunas aplicaciones industriales, debido a la baja resistencia a esfuerzos de corte, descomposición térmica, alto nivel de retrogradación y sinéresis (Bello-Pérez et al., 2002).

Por su tamaño y complejidad, el gránulo de almidón crudo no es digerible por el organismo humano debido a su estructura, por lo cual se debe utilizar un proceso de calor y humedad para transformarlo, conocido como gelatinización. Los cambios que ocurren en la gelatinización están influenciados por factores intrínsecos como tipo de almidón, tamaño de los gránulos, y por factores extrínsecos, como la velocidad de calentamiento, el 
contenido de humedad, el daño mecánico de los gránulos, la historia térmica de la muestra y las condiciones de extracción del almidón, entre otras. Para la transición se requiere un porcentaje de agua mayor al $30 \%$ y una temperatura entre 60 y $75{ }^{\circ} \mathrm{C}$, valor que depende de la fuente de origen del almidón (Pineda-Gómez et al., 2010).

El objetivo de este trabajo fue caracterizar la composición proximal, propiedades funcionales y microbiológicas de almidones de plátano cuadrado (Musa balbisiana): harina, almidón nativo, $\mathrm{AR}_{2}, \mathrm{AR}_{3} \mathrm{y}$ almidón esterilizado por calor (autoclavado), con la intención de utilizarlos como aditivos en la industria alimentaria.

\section{Materiales y Métodos}

Los frutos de plátano cuadrado (Musa balbisiana Colla) se recolectaron en plantaciones localizadas en Tabasco, México, con grado de madurez tipo I, que corresponde aproximadamente a 80 días después de la polinización. Se evaluó harina, almidón nativo, $\mathrm{AR}_{2}, \mathrm{AR}_{3} \mathrm{y}$ almidón esterilizado.

Almidones. La harina se elaboró mediante el método propuesto por da Mota et al. (2000). Para la extracción de almidón nativo se utilizó la técnica utilizada por de la Torre (2004). Los almidones resistentes $\mathrm{AR}_{2}$ y $\mathrm{AR}_{3}$ se obtuvieron mediante método enzimático (Martin et al., 1998). El $\mathrm{AR}_{3}$ se obtuvo a partir de una solución de almidón nativo de plátano al $20 \%$ y sometida al autoclave durante $30 \mathrm{~min}$ a $121^{\circ} \mathrm{C}$. El tratamiento enzimático se realizó de igual manera que para el $\mathrm{AR}_{2}$ (Lehman et al., 2002; Martin et al., 1998). El almidón autoclavado se obtuvo del almidón nativo con humedad de 9,28 \%; se introdujo en frascos de vidrio cerrados y se esterilizó en autoclave a $121^{\circ} \mathrm{C}$ durante $15 \mathrm{~min}$. Posteriormente, se mantuvo a temperatura ambiental.

Técnicas analíticas. Los análisis proximales se realizaron empleando métodos de la AOAC (1997): humedad (método 925.09), cuantificación de nitrógeno (método 954.01), extracto etéreo (método 920.39), fibra cruda (método 962.09), cenizas (método 923.03) y extracto libre de nitrógeno; por diferencia del resto de los componentes. Los patrones de solubilidad y poder de hinchamiento de los almidones se determinaron empleando el método de Sathe y Salunkhe (1981) mediante el calentamiento de una suspensión de almidón al $1 \%$ a temperaturas de 60, 70, 80 y $90{ }^{\circ} \mathrm{C}$, mediante tres repeticiones. La absorción de agua se midió por el método de Anderson et al. (1969) a temperaturas de $60,70,80$ y $90^{\circ} \mathrm{C}$, realizando tres repeticiones.

Análisis microbiológicos. La concentración de grupos bacterianos se cuantificó por el método de cultivo en superficie (Corona y Jiménez, 2004): 
aerobios mesófilos (agar Métodos Estándar, cat. 211724; BD Bioxon ${ }^{\mathrm{TM}}$ ), coliformes totales (agar Bilis Rojo Violeta, cat. 214300, BD Bioxon ${ }^{\mathrm{TM}}$ ), mohos y levaduras (agar Dextrosa y Papa, cat. 211900, BD Bioxon ${ }^{\mathrm{TM}}$ ) y Salmonella sp. (agar Salmonella-Shigella, cat. 214400, BD Bioxon ${ }^{\mathrm{TM}}$ ). Se incubaron a $37^{\circ} \mathrm{C}$ durante $24 \mathrm{~h}$.

\section{Resultados y discusión}

Caracterización proximal. El almidón de plátano es una fuente natural abundante, para lograr una aplicación exitosa en la industria es importante conocer su composición, propiedades funcionales y microbiológicas. En el Cuadro 1 se muestra la composición proximal de los almidones evaluados. En la harina y los almidones se obtuvieron productos con bajo contenido de humedad; fue en el almidón autoclavado donde se encontró la mayor concentración de humedad $(10,82 \pm 0,10)$.

Cuadro 1. Caracterización proximal del almidón de plátano cuadrado Musa balbisiana.

\begin{tabular}{lccccr}
\hline Determinación & Harina & $\begin{array}{c}\text { Almidón } \\
\text { nativo }\end{array}$ & \multicolumn{1}{c}{$A R_{2}$} & \multicolumn{1}{c}{$A R_{3}$} & \multicolumn{1}{c}{$\begin{array}{c}\text { Almidón } \\
\text { esterilizado }\end{array}$} \\
\hline Humedad & $7,45 \pm 0,28$ & $9,28 \pm 0,55$ & $8,80 \pm 0,25$ & $8,65 \pm 0,30$ & $10,82 \pm 0,10$ \\
Proteína cruda & $4,44 \pm 0,40$ & $1,19 \pm 0,04$ & $0,13 \pm 0,01$ & $0,19 \pm 0,01$ & $2,45 \pm 0,46$ \\
Extracto etéreo & $0,85 \pm 0,22$ & $0,56 \pm 0,14$ & $0,08 \pm 0,01$ & $0,42 \pm 0,05$ & $0,52 \pm 0,02$ \\
Fibra cruda & $1,88 \pm 0,16$ & $1,22 \pm 0,50$ & $0,71 \pm 0,06$ & $0,82 \pm 0,03$ & $1,21 \pm 0,36$ \\
Cenizas & $2,44 \pm 0,01$ & $0,16 \pm 0,01$ & $0,94 \pm 0,08$ & $0,50 \pm 0,05$ & $0,20 \pm 0,01$ \\
ELN & $82,94 \pm 0,21$ & $87,59 \pm 0,25$ & $89,34 \pm 0,82$ & $89,42 \pm 0,08$ & $84,80 \pm 0,37$ \\
\hline
\end{tabular}

ELN = extracto libre de nitrógeno. Resultados obtenidos de tres repeticiones.

En relación a la humedad, la harina y los almidones mostraron concentraciones entre 7,45 $\pm 0,28$ y $10,82 \pm 0,10 \%$. De acuerdo con la norma NMX-F-382-1986 para almidón o fécula de maíz, el máximo contenido de humedad permitido es de $13 \%$ (NM, 1986). En almidones de papa Pardo-C. et al. (2013) determinaron un contenido de humedad entre 5,83 y $6,90 \%$, lo cual permitió diferenciar las diversas variedades analizadas. En harina de plátano se han determinado contenidos de humedad entre 4,17 y $6,17 \%$ (da Mota et al., 2000).

La humedad es un factor importante en el almacenamiento de productos amiláceos, en harina de papa de 3 variedades, Misra y Kulshrestha (2002) analizaron el efecto del tiempo de almacenamiento en las propiedades microbiológicas, encontrando que este producto puede ser almacenado a temperatura ambiental hasta 6 meses conservando sus características. En malanga (Colocasia esculenta), se obtuvieron almidones con contenidos de humedad de 14,29 $\pm 0,50 \%$ (variedad morada) y 14,49 $\pm 0,04 \%$ (variedad blanca); estos valores se encuentran en el intervalo de humedad generalmente 
aceptado para productos secos con una vida de anaquel deseable y es menor al valor permitido $(<20 \%)$ para otros almidones convencionales (TorresRapelo et al., 2013).

En la concentración de proteínas, se observó que disminuyeron de 4,44 $\pm 0,40$ presentes en la harina hasta 1,19 $\pm 0,04$ en el almidón nativo, mientras que en los almidones resistentes su contenido fue de $0,13 \pm 0,01$ en $\mathrm{AR}_{2}$ y 0,19 $\pm 0,01$ en $\mathrm{AR}_{3}$. En el almidón esterilizado la proteína se encontró en concentración de 2,45 $\pm 0,46$; mayor a la concentración inicial en el almidón nativo. La disminución de proteínas de la harina a los almidones se relaciona con el proceso de extracción de almidón; se han evaluado soluciones de sulfato de cobre y sulfito de sodio como fijadores de proteínas, las cuales se mantienen en solución, lo que facilita su eliminación junto con el sobrenadante (Medina et al., 1990). De igual manera, el bisulfito de sodio ha sido empleado en experimentos de extracción y caracterización de almidones (HernándezMedina et al., 2008; Arzápalo et al., 2015).

El aumento de las proteínas en el almidón esterilizado probablemente esté asociado a la gelatinización del gránulo de almidón, con la posterior liberación de proteínas en su interior. Las proteínas presentes en el gránulo se encuentran unidas a su estructura interna mediante enlaces de puente de hidrógeno y disulfuro, sin embargo, estos enlaces pueden liberar fácilmente el gránulo de almidón al mantener en remojo el endospermo en una solución alcalina (Tovar-Benítez, 2008). En el maíz (Zea mays), los gránulos de almidón están embebidos en una matriz proteica que sirve como un material de soporte entre los gránulos y provee de rigidez a la estructura (NarváezGonzález et al., 2007). Debido al proceso de esterilización, los gránulos liberan proteína que puede ser cuantificable; sin embargo, desde el punto de vista industrial, la presencia de proteínas afecta las propiedades térmicas, de viscosidad y estabilidad del almidón (Pardo-C. et al., 2013).

La harina de plátano contiene valores bajos de proteína si se compara con la harina de trigo, ya que en esta última, se han determinado valores de proteína de $10 \%$ (Cho et al., 2001). Sin embargo, la proteína presente en la harina de plátano cuadrado se encontró por arriba de los obtenidos por da Mota et al. (2000) para variedades cultivadas en Brasil, donde la proteína presentó valores entre 2,5 y 3,3\%, y por Muyonga et al. (2001) para Musa canvendishii de $1,51 \%$. Las propiedades reológicas y de gelatinización en harinas y almidones son afectadas primariamente por la genética, y después de ésta, por el contenido y calidad de proteínas, porcentaje de cenizas y daño del almidón (Cho et al., 2001).

Propiedades funcionales. La solubilidad, la absorción de agua y el poder de hinchamiento son propiedades que indican la cantidad de almidón seco disuelto en agua, el volumen de agua que puede ser absorbida por gramo 
de material seco en presencia de un exceso de agua y el grado de hinchamiento de los gránulos de almidón cuando son sometidos a calentamiento durante la cocción (Jiménez-Hernández et al., 2011; Alarcón-García et al., 2013)

En relación a la solubilidad, la Fig. 1 muestra que el almidón resistente $\mathrm{AR}_{3}$ presentó mayor porcentaje en las temperaturas evaluadas, seguido por la harina integral, hasta los $80^{\circ} \mathrm{C}$. En el caso del almidón nativo, a temperaturas de 60,70 y $80{ }^{\circ} \mathrm{C}$, presentó valores de solubilidad por debajo de la harina integral, a los $90^{\circ} \mathrm{C}$, igualó la solubilidad del $\mathrm{AR}_{3}$. El almidón resistente $\mathrm{AR}_{2}$ y el esterilizado presentaron baja solubilidad; en general, la solubilidad de los almidones de plátano está directamente relacionada con el aumento de la temperatura.

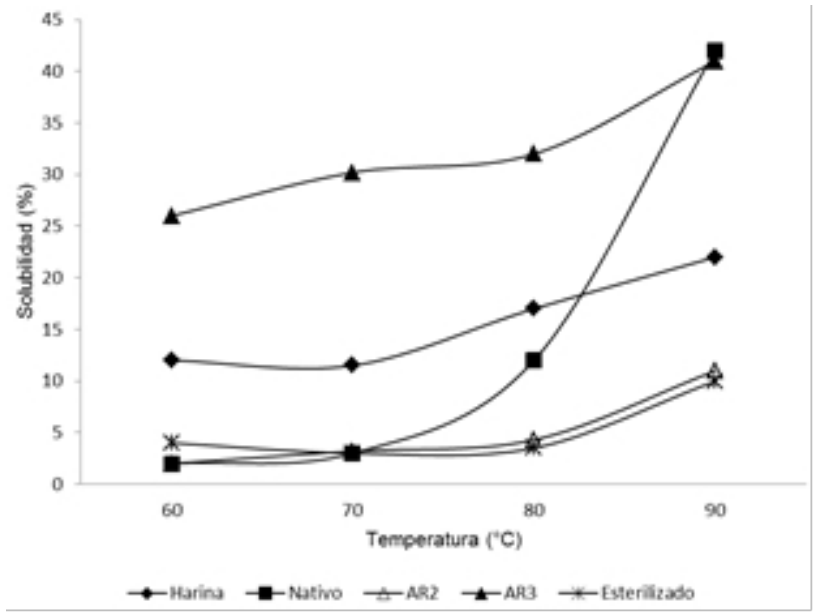

Figura 1. Solubilidad de almidones de plátano cuadrado Musa balbisiana.

De acuerdo con Ozturk et al. (2009), los valores de solubilidad de almidones autoclavados y desramificados son mayores que las de sus respectivos almidones nativos. La desramificación permite modificar la estructura de la amilopectina para generar cadenas lineales con la finalidad de formar almidones resistentes a la digestión. Se ha encontrado aumentos significativos en los valores de solubilidad con el aumento del tiempo de desramificación y los almidones desramificados presentan un mayor contenido de $\mathrm{AR}_{3}$. En almidón de plátano, se obtuvo una alta solubilidad del almidón resistente $\mathrm{AR}_{3}$.

La Fig. 2 muestra que la capacidad de absorción de agua de la harina y los almidones mostró valores bajos y sin cambios a temperaturas entre 60 y $70{ }^{\circ} \mathrm{C}$, ya que los gránulos aún no se hinchan; lo que se relaciona con una alta temperatura inicial de gelatinización. Fue la harina quien presentó la mayor capacidad de absorción de agua a los 80 y $90^{\circ} \mathrm{C}$, seguida por el almidón nativo. En relación con los almidones resistentes, el $\mathrm{AR}_{3}$ presentó su mayor capacidad de absorción de agua a los $80^{\circ} \mathrm{C}$ (colocar aquí el valor). El almidón resistente 
$\mathrm{AR}_{2}$ y el almidón esterilizado, presentaron baja capacidad de absorción de agua a bajas temperaturas; sin embargo, a $90^{\circ} \mathrm{C}$, igualaron los valores obtenidos con el $\mathrm{AR}_{3}$.

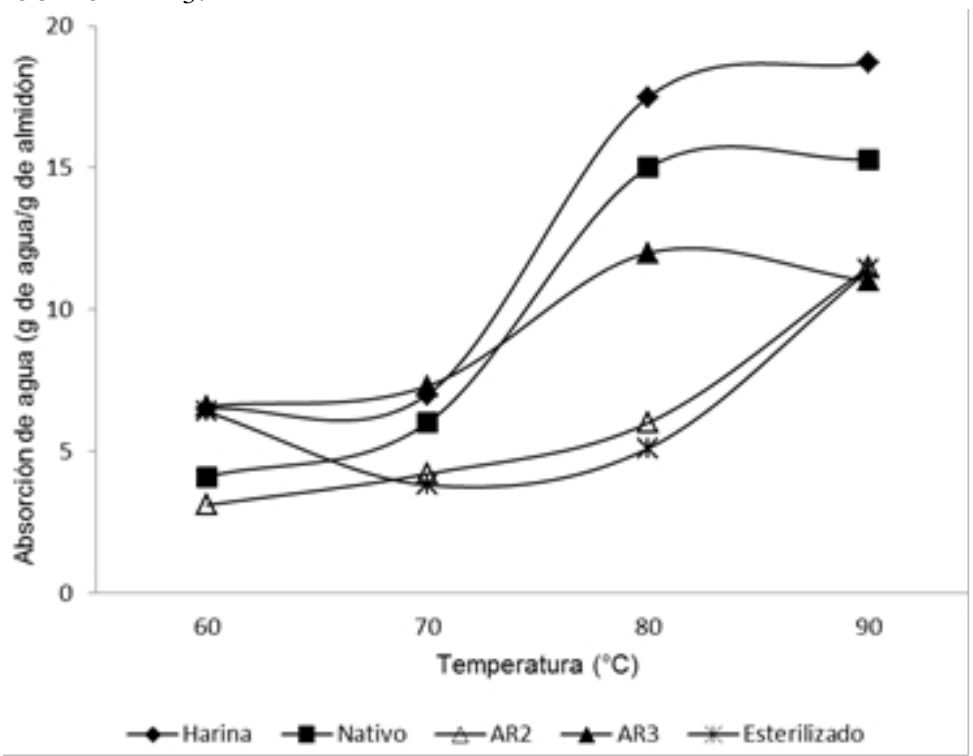

Figura 2. Capacidad de absorción de agua de almidones de plátano cuadrado Musa balbisiana.

Al comparar la capacidad de absorción de agua de los almidones de plátano cuadrado con almidones de otras fuentes, se encuentra que los almidones de plátano poseen una baja capacidad de absorción de agua. En un estudio realizado por Hernández-Medina et al. (2008) se encontró que a $90^{\circ} \mathrm{C}$ el almidón de yuca fue el que presentó mayor capacidad de absorción de agua (27,18 g de agua/g de almidón). La mayor capacidad de absorción de agua de la harina de plátano se obtuvo a los $90^{\circ} \mathrm{C}(18,7 \mathrm{~g}$ de agua/g de almidón), por debajo de la capacidad del almidón de yuca a los $90^{\circ} \mathrm{C}$.

El poder de hinchamiento es la capacidad de incremento de masa del almidón no solubilizado, debido a la absorción de agua por los grupos hidroxilo de los polímeros de amilosa y amilopectina (Meaño-Correa et al., 2014). De acuerdo a lo esperado, la capacidad de hinchamiento se incrementaó con el aumento de la temperatura, ya que a altas temperaturas se presenta una relajación progresiva de las fuerzas de enlace dentro del gránulo, lo que se traduce en un incremento del poder de hinchamiento. Esta propiedad se hizo evidente a partir de los $80{ }^{\circ} \mathrm{C}$ en el almidón nativo, harina y $\mathrm{AR}_{3}$, como se muestra en la Fig. 3. 


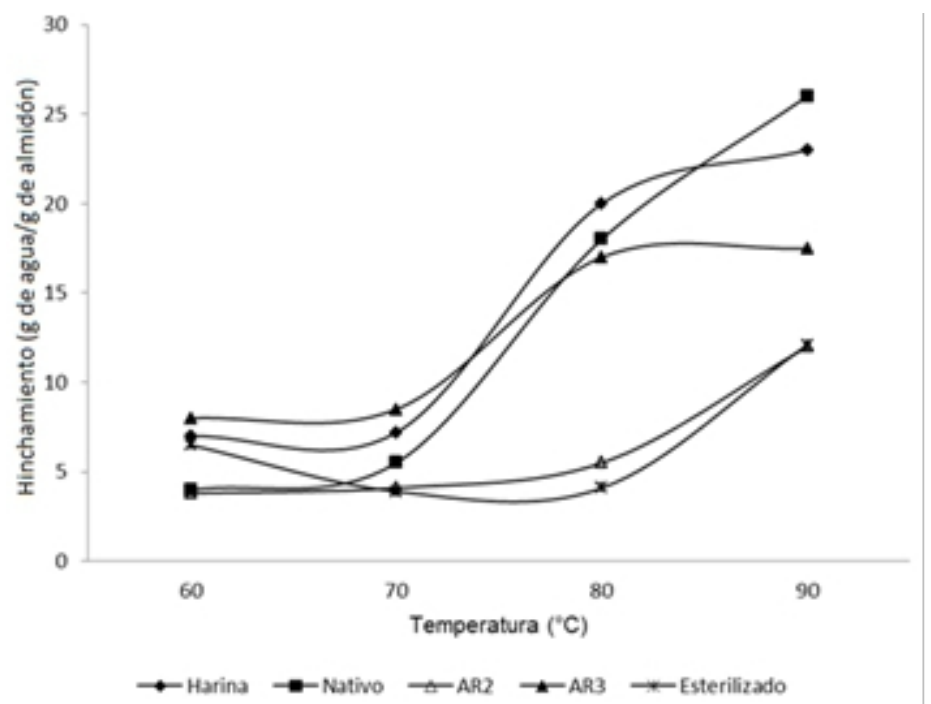

Figura 3. Poder de hinchamiento de almidones de plátano cuadrado Musa balbisiana.

En el intervalo comprendido entre 80 y $90{ }^{\circ} \mathrm{C}$, los gránulos se hinchan rápidamente a medida que se aumenta la temperatura, como resultado de la ruptura de los puentes de hidrógeno intermoleculares de las zonas amorfas, que permiten una absorción irreversible y progresiva de agua, tal como documentó Bello-Pérez et al. (1999), quienes encontraron una respuesta similar en almidones del cv. "macho". El poder de hinchamiento del almidón nativo del plátano cuadrado a $90{ }^{\circ} \mathrm{C}(26 \mathrm{~g}$ de agua/g de almidón) fue similar a los valores informados por Torruco (2004) para almidón de plátano "macho" (Musa paradisiaca L. AAB) (28 g de agua/g de almidón), pero mayor que en almidón de maíz (18 g de agua/g de almidón) (Betancur, 2001).

Estos resultados, indican que el poder de hinchamiento que presentaron los gránulos de almidón de plátano cuadrado, probablemente depende de la estructura micelar interna de los gránulos y su contenido de amilopectina. Lii et al. (1996) mencionan que el poder de hinchamiento de los almidones es una propiedad de su contenido de amilopectina, lo cual se vio reflejado en este estudio ya que el almidón de plátano puede contener bajo porcentaje de amilopectina $(68,7 \%)$. El almidón de yuca, es la especie con mayor contenido de amilopectina, como se muestra en el Cuadro 2.

Cuadro 2. Contenido de amilosa y amilopectina en almidón de diversas fuentes.

\begin{tabular}{lcc}
\hline Almidón & Amilosa (\%) & Amilopectina (\%) \\
\hline Plátano Macho & 27,0 & ND \\
Plátano Enano & 24,6 & ND \\
Plátano Morado & 31,3 & ND \\
Plátano Valery & 23,5 & ND \\
Makal & 23,6 & 76,4 \\
Camote & 19,6 & 80,4 \\
\hline
\end{tabular}




\begin{tabular}{lll}
\hline Yuca & 17,0 & 83,0 \\
Sagú & 22,7 & 77,3 \\
Maíz & 28,3 & 71,7 \\
Papa & 21,0 & 79,0 \\
\hline
\end{tabular}

Hernández-Medina et al. (2008). Méndez-Montealvo et al. (2015). ND = no documentado.

El poder de hinchamiento se relaciona con el contenido de amilopectina en el gránulo de almidón. De acuerdo con Hernández-Medina et al. (2008), el almidón de yuca fue el que presentó mayor poder de hinchamiento $\left(58,83 \mathrm{~g}\right.$ de agua/g de almidón a $\left.90^{\circ} \mathrm{C}\right)$, con $17 \%$ de amilosa. El makal presentó 28,56 g de agua/g de almidón, con 23,6 \% de amilosa; el camote 25,53 g de agua/g de almidón, con 19,6 \% de amilosa y, finalmente, el sagú 16,98 g de agua/g de almidón, con 22,69 \% de amilosa. En el presente experimento se obtuvieron $26,0 \mathrm{~g}$ de agua/g de almidón en almidón nativo de plátano, con contenidos de amilosa entre 23,5 y $31,3 \%$, de acuerdo a lo documentado por Méndez-Montealvo et al. (2015).

Este comportamiento funcional de los almidones de plátano también pudo deberse al tamaño de sus gránulos y, probablemente, a la estructura más ramificada de la amilopectina, en la cual hay una mayor interacción con la molécula de agua (Bello-Pérez, 1995). En este sentido, los gránulos del almidón de plátano cuadrado presentan un diámetro promedio de $24,61 \mu \mathrm{m}$, mayor al diámetro de gránulos de mango, makal y yuca, como se muestra en el Cuadro 3 (Jiménez-Vera et al., 2011).

Cuadro 3. Forma y tamaño de los gránulos de almidón nativo en diversas fuentes.

\begin{tabular}{llcc}
\hline \multicolumn{1}{c}{ Fuente } & \multicolumn{1}{c}{ Forma } & Diámetro promedio $(\mu \mathrm{m})$ & Intervalo $(\mu \mathrm{m})$ \\
\hline Plátano cuadrado & Oval, alargada & 24,61 & $8-68$ \\
Plátano macho & Oval, alargada & ND & $20-50$ \\
Mango & Oval, alargada & 15,80 & $6,5-20,7$ \\
Frijol x'pelón & Oval, lisa & 17.90 & $11,8-26,7$ \\
Frijol ib & Oval, esférica & 23,60 & $12-45$ \\
Makal & Esférica & 12,4 & $8-20$ \\
Yuca & Semiesfera & ND & $8-17$ \\
\hline
\end{tabular}

$\mathrm{ND}=$ no determinado. Jiménez-Vera et al. (2011).

El comportamiento en las propiedades funcionales del almidón esterilizado fue similar a las del almidón resistente $\mathrm{AR}_{2}$, ambos presentaron valores bajos de solubilidad, absorción de agua y poder de hinchamiento. La esterilización del almidón nativo a baja humedad permitió obtener un almidón con una mejor calidad microbiológica; sin embargo, se encontró una disminución de sus propiedades funcionales. La harina, el almidón nativo y el almidón $\mathrm{AR}_{3}$, son una opción para el uso funcional de almidones en alimentos sometidos a temperaturas mayores a $\operatorname{los} 90^{\circ} \mathrm{C}$ y con un elevado contenido de 
humedad, donde se requiere la absorción de agua y el poder de hinchamiento del almidón.

De acuerdo con Reddy et al. (2013), la industria alimentaria requiere almidones con buena capacidad de absorción de agua, solubilidad y cristalinidad junto con mayor estabilidad térmica. Estos investigadores analizaron las propiedades funcionales del almidón resistente de frijol rojo (Phaseolus vulgaris L.), donde obtuvieron una alta capacidad de absorción de agua y solubilidad, pero baja viscosidad. La viscosidad fue inversamente proporcional al contenido de almidón resistente.

Los almidones resistentes poseen propiedades fisicoquímicas deseables como poder de hinchamiento, aumento de la viscosidad, formación de gel y capacidad de retención de agua, por lo que pueden incluirse en una gran variedad de alimentos. Su baja capacidad de retención de agua los convierte en ingredientes funcionales que proporcionan un buen manejo y mejoras en la textura en el producto final. Con algunos almidones resistentes es posible obtener una mejor sensación en la boca, color y sabor que la obtenida con ciertas fibras insolubles tradicionales. Esto aumenta la posibilidad que los consumidores acepten estos alimentos y, por lo tanto, incrementen su ingesta de fibra dietética. Estas propiedades de los almidones resistentes permiten reemplazar la harina en una proporción 1:1, sin afectar la manipulación de la masa o su reología (Fuentes-Zaragoza et al., 2010).

El almidón resistente muestra efectos fisiológicos similares a la fibra soluble. Los alimentos con mayor cantidad de almidón resistente contienen menos calorías y cargas glucémicas inferiores, lo cual es importante para los diabéticos y para las personas conscientes del peso. Es factible aumentar los contenidos de almidón resistente en los alimentos al cambiar las condiciones de procesamiento como el $\mathrm{pH}$, el tiempo, la temperatura, el número de ciclos de calentamiento y enfriamiento, la congelación y el secado (Ashraf et al., 2012).

Calidad microbiológica. Los resultados en las cuentas microbianas se muestran en el Cuadro 4. En el almidón nativo se encontró el mayor número de microorganismos mesófilos y coliformes. En almidón $\mathrm{AR}_{3}$, debido a la esterilización en su proceso, el valor de coliformes totales fue negativo, al igual que el almidón esterilizado. En ningún almidón se encontró Salmonella sp.

Cuadro 4. Calidad microbiológica de almidones y harina de plátano cuadrado.

\begin{tabular}{lccccc}
\hline $\begin{array}{c}\text { Microorganismos } \\
(\log \text { UFC/g) }\end{array}$ & Harina & $\begin{array}{c}\text { Almidón } \\
\text { nativo }\end{array}$ & $\mathrm{AR}_{2}$ & $\mathrm{AR}_{3}$ & $\begin{array}{c}\text { Almidón } \\
\text { esterilizado }\end{array}$ \\
\hline Mesófilos aerobios & $5,43 \pm 0,46$ & $7,99 \pm 0,09$ & $6,02 \pm 0,13$ & $5,66 \pm 0,10$ & $\mathrm{ND}$ \\
Coliformes & $3,93 \pm 0,35$ & $4,17 \pm 0,23$ & $3,79 \pm 0,50$ & $\mathrm{ND}$ & $\mathrm{ND}$ \\
\hline
\end{tabular}




\begin{tabular}{lccccc}
\hline Hongos & $4,54 \pm 0,34$ & $7,45 \pm 0,21$ & $5,54 \pm 0,34$ & $4,45 \pm 0,21$ & ND \\
Levaduras & $3,83 \pm 0,50$ & $8,30 \pm 0,30$ & $5,58 \pm 0,49$ & $4,39 \pm 0,36$ & ND \\
Salmonella sp. & ND & ND & ND & ND & ND \\
\hline
\end{tabular}

$\mathrm{ND}=$ no detectable.

En el proceso de extracción de almidón las temperaturas empleadas en el secado son bajas $\left(55^{\circ} \mathrm{C}\right)$ para no dañar al gránulo de almidón, lo que favorece el crecimiento de microorganismos. La esterilización asegura la ausencia de microorganismos, sin embargo, genera efectos sobre la estructura de los gránulos de almidón al gelatinizarse en presencia de agua y calor. Una alternativa para evitar la gelatinización de los gránulos de almidón y lograr buena calidad microbiológica es la esterilización a bajo contenido de humedad. No obstante, la esterilización tiene efectos negativos en las propiedades funcionales del almidón nativo.

\section{Conclusiones y Recomendaciones}

Se determinó la composición proximal, propiedades funcionales y calidad microbiológica de almidones de plátano cuadrado (Musa balbisiana). La concentración de proteínas se relaciona con la calidad de la extracción del almidón, la mayor concentración se obtuvo en harina $(4,44 \pm 0,40)$ y la menor en almidón nativo $(1,19 \pm 0,04)$.

En cuanto a las propiedades funcionales, la mayor solubilidad se obtuvo con el almidón nativo (42\%) y el almidón resistente tipo III (41 \%), a $90{ }^{\circ} \mathrm{C}$; el mayor poder de hinchamiento lo presentó el almidón nativo (26 g de agua/g de almidón) y la harina ( $23 \mathrm{~g}$ de agua/g de almidón), mientras que en la absorción de agua fue la harina ( $18.7 \mathrm{~g}$ de agua/g de almidón) y el almidón nativo ( $15.3 \mathrm{~g}$ de agua/g de almidón) los que presentaron mayor absorción, a $90{ }^{\circ} \mathrm{C}$.

El almidón nativo presentó la mayor contaminación microbiológica: mesófilos aerobios $(7,99 \pm 0,09 \log \mathrm{UFC} / \mathrm{g})$, coliformes $(4,17 \pm 0,23 \mathrm{log}$ UFC/g), hongos (7,45 $\pm 0,21 \log$ UFC/g) y levaduras $(8,30 \pm 0,30 \log \mathrm{UFC} / \mathrm{g})$. El almidón resistente tipo III presentó las mejores propiedades funcionales y una buena calidad microbiológica, por lo que se recomienda su evaluación como ingrediente de alimentos funcionales. Aunque la calidad microbiológica del almidón esterilizado fue la mejor, sus propiedades funcionales, junto con el almidón resistente tipo II, fueron las que presentaron los valores más bajos. Se recomienda evaluar el almidón de plátano en alimentos que requieran gran solubilidad y mediano poder de hinchamiento con la finalidad de disminuir la ingesta de calorías e incrementar el valor de fibra dietética soluble. 


\section{References:}

1. Alarcón-García, Miguel Ángel; López-Vargas, Jairo Humberto y Restrepo-Molina, Diego Alonso. 2013. Caracterización de la funcionalidad tecnológica de una fuente rica en fibra dietaria obtenida a partir de cáscara de plátano. Revista Facultad Nacional de Agronomía, Medellín. 66(1):6959-6968.

2. Anderson, R., Conway, H., Pfeifer, V. y Griffin, E. (1969). Gelatinization of corn grits by roll and extrusion cooking. Cereal Sci. Today. 14:4-12.

3. AOAC. 1997. Association of Official Analytical Chemist. Official Methods of Analysis. (17ta. ed.). Washington, D. C., USA.

4. Arzapalo, D., Huamán, K., Quispe, M. y Espinoza, C. 2015. Extracción y caracterización del almidón de tres variedades de quinua (Chenopodium quinoa Willd) negra Collana, Pasankalla roja y blanca Junín. Rev Soc Quím Perú. 81(1):44-56.

5. Ashraf, Saira; Anjum, Faqir Muhammad; Nadeem, Muhammad and Riaz, Asad. 2012. Functional \& technological aspects of resistant starch. Pakistan Journal of Food Sciences. 22(2):90-95.

6. Bello-Pérez, L. 1995. Amilopectina: caracterización molecular y funcional. Tesis Doctoral. Centro de Investigación y de Estudios Avanzados del Instituto Politécnico Nacional, Unidad Irapuato, Guanajuato, México. pp. 81-122.

7. Bello-Pérez, Luis A.; Agama-Acevedo, Edith; Sánchez-Hernández, Laura and Paredes-López, Octavio. 1999. Isolation and partial characterization of banana starches. Journal of Agricultural and Food Chemistry. 47(3):854-857.

8. Bello-Pérez, Luis Arturo; Contreras-Ramos, Silvia Maribel; RomeroManilla, Rhebeca; Solorza-Feria, Javier y Jiménez-Aparicio, Antonio. 2002. Propiedades químicas y funcionales del almidón modificado de plátano Musa paradisiaca L. (var. Macho). Agrociencia. 36(2):169180.

9. Betancur, D. 2001. Caracterización molecular, nutricia y funcional de almidones de Phaseolus lunatus y Mucna pruriens. Tesis Doctoral. Instituto Politécnico Nacional, México, D. F. pp. 10-21, 33-38.

10. Blasco-López, Gabriela y Gómez-Montaño, Francisco Javier. 2014. Propiedades funcionales del plátano (Musa sp.). Revista Médica de la Universidad Veracruzana. 14(2):22-26.

11. Corona, A. y Jiménez, R. 2004. Comparación de dos métodos de siembra para el recuento de microorganismos en muestras con alta concentración microbiana. Revista de la Facultad de Ingeniería Química. 40:3-7. 
12. Cho, S.Y.; Tak, S.H. and Rhee, Ch. 2001. Effect of extraction rate of korean wheat flour on rheological and raw noodle-making properties. Food Science and Biotechnology (Corea). 10(3):246-250.

13. da Mota, Renata Vieira; Lajolo Franco M.; Cordenunsi, Beatriz R. and Ciacco, Cesar. 2000. Composition and functional properties of banana flour from different varieties. Starch. 52(2-3):63-68.

14. de la Torre, L. 2004. Extracción y caracterización fisicoquímica y funcional del almidón de plátano cuadrado (Musa balbisiana Colla). Tesis de Maestría. Facultad de Ingeniería Química (FIQ), Universidad Autónoma de Yucatán (UADY), México. pp 35.

15. Fuentes-Zaragoza, E.; Riquelme-Navarrete, M.J.; Sánchez-Zapata, E. and Pérez-Álvarez, J.A. 2010. Resistant starch as functional ingredient: a review. Food Research International. 43(4):931-942.

16. Hernández-Medina, Marilyn; Torruco-Uco, Juan Gabriel; ChelGuerrero, Luis y Betancur-Ancona, David. 2008. Caracterización fisicoquímica de almidones de tubérculos cultivados en Yucatán, México. Ciência e Tecnologia de Alimentos. 28(3):718-726.

17. Jiménez-Hernández, J.; Meneses-Esparza, F.; Rosendo-Escobar, J.; Vivar-Vera, M.A.; Bello-Pérez, L.A. y García-Suárez, F.J. 2011. Extracción y caracterización del almidón de las semillas de Enterolobium cyclocarpum. CyTA - Journal of Food. 9(2):89-95.

18. Jiménez-Vera, Román; González-Cortés, Nicolás; Magaña-Contreras, Arturo y Corona-Cruz, Alma Irene. 2011. Contenido de almidón resistente en alimentos consumidos en el sureste de México. UNACAR Tecnociencia. 5(2):27-34.

19. Langkilde, Anna Maria; Champ, Martine y Andersson, Henrik. 2002. Effects of high-resistant-starch banana flour $\left(\mathrm{RS}_{2}\right)$ on in vitro fermentation and the small-bowel excretion of energy, nutrients, and sterols: an ileostomy study. The American Journal Clinical Nutrition. 75(1):104-111.

20. Lehman, Undine; Jacobasch, Gisela and Schmiedl, Detlef. 2002. Characterization of resistant starch type III from banana (Musa acuminata). Journal of Agricultural and Food Chemistry. 50(18):52365240.

21. Lii, Ch. Y.; Tsai, M.L. and Tseng, K.H. 1996. Effect of amylose content on the rheological property of rice starch. Cereal Chemistry. 73(4):415-420.

22. Lockyer, S. and Nugent, A.P. 2017. Health effects of resistant starch. Nutrition Bulletin. 42(1):10-41.

23. Martin, Lucile J.M. ; Dumon, Henri J.W. and Champ, Martine M.J. 1998. Production of short-chain fatty acids from resistant starch in a 
pig model. Journal of the Science of Food and Agriculture. 77(1):7180.

24. Meaño-Correa, Ninoska; Ciarfella-Pérez, Ana Teresa y DortaVillegas, Ana Marina. 2014. Evaluación de las propiedades químicas y funcionales del almidón nativo de ñame congo (Dioscorea bulbifera L.) para predecir sus posibles usos tecnológicos Saber. 26(2):182-187.

25. Medina, L., Mackenzie, J. y Bermudez, A. 1990. Obtención de almidón bajo en proteínas a partir de maíz desgerminado. Revista Colombiana de Química. 19(2)07-14.

26. Méndez-Montealvo, G.; Rodriguez-Ambriz, S.L. and Bello-Pérez, L.A. 2015. Structural features of banana starches using HPSECMALLS-RI. Revista Mexicana de Ingeniería Química. 14(2):293-302.

27. Misra, Anupama and Kulshrestha, Kalpana. 2002. Effect of storage on microbial safety of potato flour. Journal of Food Science and Technology. 39(5):517-519.

28. Muyonga, J.H.; Ramteke, R.S. and Eipeson, W.E. 2001. Predehydration steaming changes physicochemical properties of unripe banana flour. Journal of Food Processing and Preservation. 25(1):35-47.

29. Narváez-González, Ernesto David; Figueroa-Cárdenas, Juan de Dios; Taba, Suketoshi; Castaño-Tostado, Eduardo y Martínez-Peniche, Ramón Álvar. 2007. Efecto del tamaño del gránulo de almidón de maíz en sus propiedades térmicas y de pastificado. Revista Fitotecnia Mexicana. 30(3):269-277.

30. NM. 1986. Norma Mexicana NMX-F-382-1986. Alimentos. Almidón o fécula de maíz.

31. Olvera-Hernández, V.; Aparicio-Trápala, M.A.; Ble-Castillo, J.L.; Muñoz-Cano, J.M. y Rodríguez-Blanco, L. 2012. Efecto del almidón resistente de banano (Musa cavendish AAA) sobre el control metabólico en ratas Wistar con dieta alta en sacarosa. Universidad y Ciencia. 28(1):51-56.

32. Ozturk, Serpil; Koksel, Hamit; Kahraman, Kevser and Ng, Perry K.W. 2009. Effect of debranching and heat treatments on formation and functional properties of resistant starch from high-amylose corn starches. European Food Research and Technology. 229(1):115-125.

33. Parada-S., Javier Alejandro y Rozowski-N., Jaime. 2008. Relación entre la respuesta glicémica del almidón y su estado microestructural. Revista Chilena de Nutrición. 35(2):84-92.

34. Pardo-C., Oscar Hernando; Castañeda, Julio César y Ortiz, César Armando. 2013. Caracterización estructural y térmica de almidones provenientes de diferentes variedades de papa. Acta Agronómica. 62(4):289-295. 
35. Pineda-Gómez, P.; Coral, D.F.; Arciniegas, M.L.; Rorales-Rivera, A. y Rodríguez-García, M.E. 2010. Papel del agua en la gelatinización del almidón de maíz: estudio por calorimetría diferencial de barrido. Ingeniería y Ciencia. 6(11):129-141.

36. Reddy, Chagam Koteswara; Suriya, M. and Haripriya, Sundaramoorthy. 2013. Physico-chemical and functional properties of resistant starch prepared from red kidney beans (Phaseolus vulgaris L.) starch by enzymatic method. Carbohydrate Polymers. 95(1):220226.

37. Rodríguez-Aguilar, D.; Ariza-Nieto, C.J. y Afanador-Téllez, G. 2014. Potencial del almidón resistente retrogradado de papa frente a otros aditivos funcionales usados en pollos de engorde. Revista de la Facultad de Medicina Veterinaria y de Zootecnia. 61(1):44-63.

38. Sathe, S.K. and Salunkhe, D.K., 1981. Isolation, partial characterization and modification of the Great Northern bean (Phaseolus vulgaris) starch. Journal of Food Science. 46(2):617-621.

39. Singhal, R.S. and Kulkarni, P.R. 1988. Amaranths-an underutilized resource. International Journal of Food Science and Technology. 23(2):125-139.

40. Soto-Azurduy, Vania Soraya. 2010. Cuantificación de almidón total y de almidón resistente en harina de plátano verde (Musa cavendishii) y banana verde (Musa paradisiaca). Revista Boliviana de Química. 27(2):94-99.

41. Torres-Rapelo, Alberto; Montero Castillo, Piedad y Duran-Lengua, Marlene. 2013. Propiedades fisicoquímicas, morfológicas y funcionales del almidón de malanga (Colocasia esculenta). Revista Lasallista de Investigación. 10(2):52-61.

42. Torruco, J. 2004. Estructura y funcionalidad de almidones de makal (Xanthosoma yucatenensis Stnadl), frijol x'pelón (Vigna unguiculata L.) y plátano macho (Musa paradisiaca L. AAB). Tesis de Maestría. Facultad de Ingeniería Química, Universidad Autónoma de Yucatán, Mérida, Yucatán, México.

43. Valerio-Dávila, Frecia. 2014. Determinación de los parámetros para la extracción de almidón del plátano bellaco (Musa paradisiaca). Revista de Investigación Universitaria. 3(2):23-28. 